\title{
A CASE OF SCRUB TYPHUS FEVER PRESENTING WITH MULTI-ORGAN FAILURE
}

\author{
Sudhir Kumar1, Govind Kumar², Jyoti Kumar Dinkar³, Mohit Kumar Jaiswal4, Jyoti Gautam 5
}

${ }^{1}$ Additional Professor, Department of General Medicine, IGIMS, Patna. ${ }^{2}$ Additional Professor, Department of General Medicine, IGIMS, Patna. 3 Senior Resident, Department of General Medicine, IGIMS, Patna. ${ }^{4}$ Senior Resident, Department of General Medicine, IGIMS, Patna. ${ }^{5}$ Senior Resident, Department of General Medicine, IGIMS, Patna.

HOW TO CITE THIS ARTICLE: Kumar S, Kumar G, Dinkar JK, et al. A case of scrub typhus fever presenting with multi- organ failure. J. Evolution Med. Dent. Sci. 2018;7(07):942-943, DOI: 10.14260/jemds/2018/215

\section{PRESENTATION OF CASE}

A 25-year-old female patient with no previous co-morbidities came to General Medicine department of IGIMS, Patna, Bihar with presenting complaints of fever associated with chill and rigor for a duration of 15 days, yellowish discoloration of eyes and urine for a duration of 12 days, decrease urine output for 5 days and constipation for 3 days. She had no previous history of hypertension, diabetes mellitus, tuberculosis, asthma, jaundice and drug abuse. She had history of pain abdomen around peri-umbilical region and pain in bilateral lower limb which subsided after treatment in the periphery by local doctor. She had no history of preceding systemic symptoms prior to the present illness. She had consulted doctor earlier in periphery who referred her to higher centre. On examination of the patient with consent she was found to be conscious, oriented and febrile. Icterus was present but there was no pallor, lymphadenopathy or oedema. On systemic examination, respiratory, cardiovascular, gastrointestinal and central nervous systems appeared to be normal. At the time of admission, she was febrile with pulse rate $108 \mathrm{bpm}, \mathrm{BP} 120 / 70 \mathrm{mmHg}$, respiratory rate $20 / \mathrm{min}$. There was no rash over the exposed skin.

Although India lies in geographical area known as tsutsugamushi triangle, it is generally under-diagnosed in Bihar because of low index of suspicion and poor diagnostic backup. Scrub typhus presentation ranges from mild illness to severe multi-organ system involvement. We are reporting a case of scrub typhus presenting as multi-organ failure who was diagnosed promptly and treated successfully.

\section{DIFFERENTIAL DIAGNOSIS}

After considering initial clinical presentation of the patient possibilities of malaria, enteric fever, dengue fever, acute viral hepatitis, acute renal failure, urinary tract infection were all looked into. The complete blood count, antigen test for malaria, Widal test, serum electrolytes, ANA, ECG, chest x-ray PA view were normal. Liver function test report revealed raised total bilirubin $(2.03 \mathrm{mg} / \mathrm{dl})$, conjugated bilirubin $(1.88$ $\mathrm{mg} / \mathrm{dl})$, and SGPT (849 U/l) although SGOT level was on upper limit of normal. Blood urea and serum creatinine were

'Financial or Other Competing Interest': None.

Submission 24-12-2017, Peer Review 26-01-2018,

Acceptance 01-02-2018, Published 12-02-2018.

Corresponding Author:

Jyoti Kumar Dinkar,

Flat 101, Block-A, Ramgati Residency,

Ashiyana Nagar, Near Bandhan Bank,

Patna-800025, Bihar.

E-mail:drdinkar03@gmail.com

DOI: $10.14260 /$ jemds $/ 2018 / 215$

\section{(c) $($ ) $\$$}

56 and $3.1 \mathrm{mg} / \mathrm{dl}$ respectively. Urine culture report showed no growth with few pus cells on microscopy. Patient had no urinary symptoms. Ultrasonography of abdomen suggests acute medical renal disease with mild ascites. Dengue serology, Elisa for HbsAg, anti HCV, HIV serology, anti HAV IgM and anti HEV IgM were all negative.

\section{CLINICAL DIAGNOSIS}

Despite giving empirical antimalarial agents and antibiotics, patient condition deteriorated day by day with involvement of multiple organs. Although not very common but suspicion of scrub typhus fever and leptospirosis was made as patient presented in monsoon season and was from rural area. On meticulous search, a skin lesion with black scab i.e. eschar was found on the arm near to axillary area [Photograph-1] Scrub typhus fever may present with multi-organ failure. However, it is not necessary to have an eschar in each and every case of scrub typhus so we sent the patient's blood sample for IgM serology for scrub typhus and leptospira. The report revealed positive for scrub typhus and negative for leptospira. So, a diagnosis of scrub typhus with multiorgan failure was made.

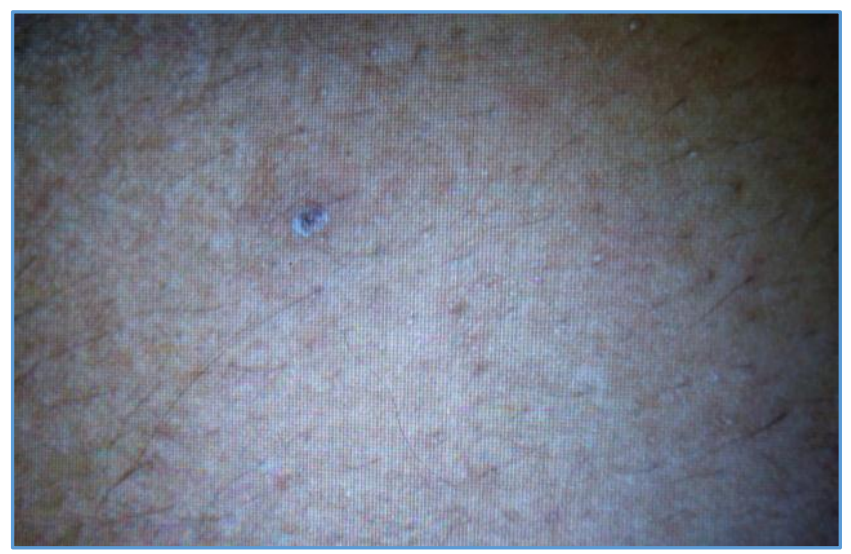

Photograph 1. Eschar found on the arm near the axillary region of the patient

\section{PATHOLOGICAL DISCUSSION}

Scrub typhus is an acute febrile illness caused by the organism Orientia tsutsugamushi an obligate intracellular gram-negative coccobacillus. The name is derived from the vegetation type that usually harbours the vectors. It lacks lipopolysaccharides in its cell wall. It is transmitted by the bite of infected larvae of trombiculid mites (chiggers) and maintained by transovarial transmission. Illness varies from mild and self-limiting to fatal in rare cases with an incubation period of 6-21 days. Disease onset is characterised by fever, headache, myalgia, cough and gastro intestinal symptoms. Some patients recover spontaneously after a few days and 
rarely presents with multiorgan failure. The classic case description includes an eschar where the chigger has fed, regional lymphadenopathy and a maculopapular rash, signs that are seldom seen in indigenous patients.

Scrub typhus is endemic to a distinct region, the tsutsugamushi triangle, which include Japan, Taiwan, China and South Korea.[1] Scrub typhus is known to occur all over India including the north hilly areas.[2,3,4] Cases of scrub typhus from state of Bihar are few and that too reported from hospitals of other states. Fever is most important feature of scrub typhus and it is considered as one of the causes of "Pyrexia of unknown origin".[5] The clinical manifestations may vary from sub-clinical disease to organ failure to fatal disease.[6] Scrub typhus was first described by Hashimoto from Japan[7]. When man encroaches the mite-infested area he is prone to get infected accidentally. Scrub growth especially the secondary scrub growth occurs after clearance of primary forest. Cases are more common in rainy season. The chigger phase of mite gets attached to skin of host and prefers to feed where skin is thin and wrinkled. Large number of organism are injected into its host when it feeds. In many patients, skin lesion, black eschar, develop at the site of chigger attachment. It is one of the clinical diagnostic clue of scrub typhus.

Scrub typhus has a broad clinical spectrum from mild selflimiting illness to multi-organ failure. This disease is underdiagnosed in Bihar because of very low index of suspicion and lack of diagnostic facilities. In scrub typhus, complications usually develop after few days of febrile illness. Jaundice, renal failure, pneumonitis, ARDS, septic shock, myocarditis and meningo-encephalitis are various complications known with this disease.[2,8]

Early diagnosis is essential to cut the morbidity and mortality in scrub typhus. Lab diagnosis is based on mainly serological tests. Weil Felix has low sensitivity but useful in areas where other serological tests are not available. Indirect fluorescent antibody test and ELISA are better serological tests. Tetracycline, and Doxycycline are treatment of choice. Azithromycin has been proved more effective than Doxycycline in both Doxycycline-susceptible and Doxycycline-resistant strains causing scrub typhus.[9] Resistance to tetracycline has been noted in few areas.[10]

In this case, patient presented with multi-organ involvement. An early suspicion lead to diagnosis and initiation of proper treatment. This patient responded and recovered from illness.

\section{DISCUSSION OF MANAGEMENT}

In view of the patient's clinical deterioration despite empirical antimalarial and antibiotics and positive IgM serology for scrub typhus, we put the patient on Tab Doxycycline $100 \mathrm{mg}$ bid orally and other symptomatic treatment. Patient started to recover from day 5 of initiation of oral Doxycycline. It is clear from this case that although scrub typhus fever varies from mild to self-limiting course, it can also present with acute febrile illness with multiorgan dysfunction. So, it is better to have high index of suspicion for scrub typhus in such types of presentation. We discharged the patient on day 10 after normalisation of haematological and biochemical parameters and advised her to come for follow up after 7 days in Medicine OPD. She came for follow up and is doing well.

\section{FINAL DIAGNOSIS}

Based on the clinical presentation and investigation a final diagnosis of scrub typhus fever with multiorgan failure was confirmed and patient responded well to the treatment.

\section{REFERENCES}

[1] Chang WH. Current status of tsutsugamushi disease in korea. J Korean Med Sci 1995;10(4):227-38.

[2] Cowan GO. Rickettsial infections. In: Manson's tropical diseases. Gordon C. (eds). 21 st edn. London Saunders Elsevier Science. Health Sciences Division. 2003;50:891-906.

[3] Watt G. Scrub typhus In Oxford textbook of medicine. Warrel DA, Cox TM, Firth JD, et al. (eds). $4^{\text {th }}$ edn. Oxford. Oxford University Press, 2003;1:629-31.

[4] Walker D, Raolt D, Dumler JS, et al. In Harrison's principles of internal medicine. Braunwald E, Fauci AS, Kasper DL, et al. (eds). 15 th edn. New York McGraw Hill Companies Inc. 2001;1:1070.

[5] Yang CH, Hsu GJ, Peng MY, et al. Hepatic dysfunction in scrub typhus. J Formos Med Assoc 1995;94(3):101-5.

[6] Walsh DS, Myint KS, Kantipong P, et al. Orientia tsutsugamusi in peripheral white blood cells of patients with acute scrub typhus. Am J Trop Med Hyg 2001;65(6):899-901.

[7] Mahajan SK. Scrub typhus. J Assoc Physician India 2005;53:954-8.

[8] Silpapojakul K. Scrub typhus in Western Pacific region. Ann Acad Med Singapore 1997;26(6):794-800.

[9] Strickman D, Sheer T, Salata K, et al. In vitro effectiveness of azithromycin against doxycyclineresistant and -susceptible strains of Rickettsia tsutsugamushi, etiological agent of scrub typhus. Antimicrob Agents Chemother 1995;39(11):2406-10.

[10] Watt G, Chouriyagune C, Ruangweerray T, et al. Scrub typhus infections poorly responsive to antibiotics in northern Thailand. Lancet 1996;348(9020):86-9. 